\title{
Modeling of light propagation in photonic liquid crystal fibers
}

\author{
Katarzyna A. Rutkowska* and Tomasz R. Woliński \\ Faculty of Physics, Warsaw University of Technology, Koszykowa 75, 00-662 Warszawa, Poland
}

Received September 20, 2010; accepted September 29, 2010; published September 30, 2010

\begin{abstract}
In this letter, a numerical analysis of light propagation in photonic crystal fibers infiltrated with liquid crystals is presented. Our simulations, based on the finite difference beam propagation method, revealed the importance of space discretization in numerical algorithms applied. At the conclusion, a triangular discrete mesh is shown as better numerical representation when compared to the standard square grid used for modeling photonic crystal fibers with hexagonal symmetry.
\end{abstract}

Photonic crystal fibers (PCFs), usually formed by silica glass and air-holes periodically distributed therein [1], are an important subject for scientific investigation, arising mainly from their particular optical properties and a great number of potential applications. On the way of development of novel photonic elements and devices, special interest has been dedicated to PCFs infiltrated with different substances and materials. In this context, the choice of nematic liquid crystals (NLCs) as practical and functional infiltration has been motivated by the variety of available mixtures with different chemical, physical and optical properties, inexpensive and consolidated fabrication technology, considerable thermal and electric responses, extended spectral transparency, huge birefringence, giant reorientational nonlinearity, and many others [2]. As a result, a new class of advanced microstructures, called photonic liquid crystal fibers (PLCFs), has been established, allowing for the creation of flexible and tunable photonic devices [3-6].

As it is well-known, PLCFs can guide light in the glass core (e.g. due to the existence of the photonic bandgap), as well as in the region of infiltrated holes, and moreover, the mechanism of light propagation can be switched by modification of NLC refractive indices [7]. The latter may be achieved thermally, by applying external (electric or magnetic) fields, or by increasing optical power [8-9]. In particular, when the refractive index of an infiltrating material is higher than the one of the silica surround, the analyzed PLCFs with a spatial periodicity of refractive index distribution may be considered as a matrix of waveguide channels. This architecture, analogical to a multi-core optical fiber, allows for discrete light propagation [8-10].

\footnotetext{
"E-mail: kasia@if.pw.edu.pl
}

(a)

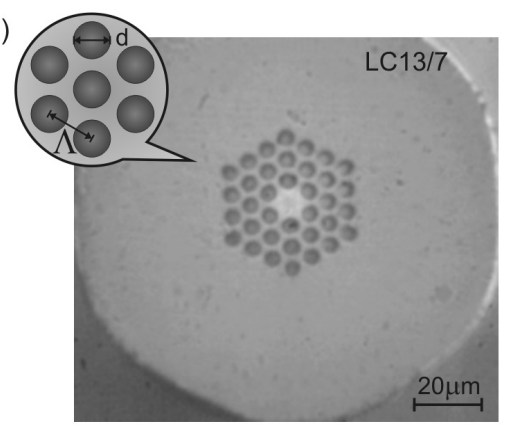

(b)

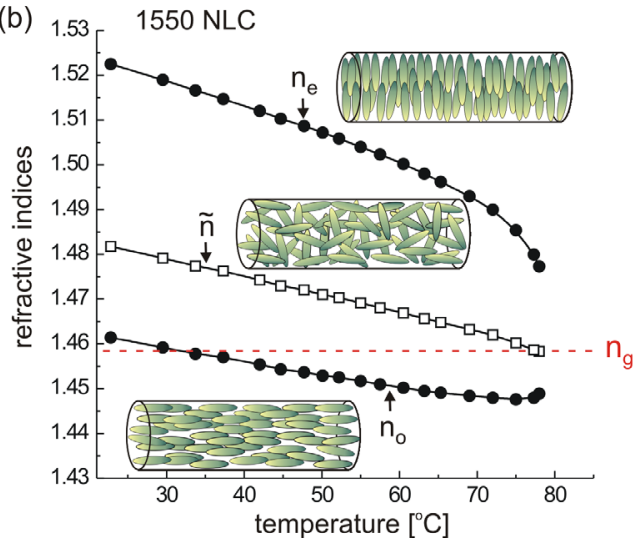

Fig. 1. (a) Picture of the cross-section of an analyzed PCF, where $d$ is a hole diameter and $\Lambda$ is a distance between neighbouring holes (i.e. pitch of periodic structure). (b) Temperature dependence of the 1550 NLC refractive indices, where $n_{o}$ is an ordinary and $n_{e}-$ an extraordinary refractive index. The average value of the refractive index (corresponding to disordered (isotropic) phase) is calculated as: $\tilde{\mathrm{n}}=2 \mathrm{n}_{\mathrm{o}} / 3+\mathrm{n}_{\mathrm{e}} / 3$ [2]. The refractive index of silica glass $\mathrm{n}_{\mathrm{g}}$ is also presented (red dashed line) [11].

A cross-section picture of the PCF structure under investigation is shown in Fig. 1a. This specific host fiber, called LC13/7 and fabricated at the Maria Curie Skłodowska University in Lublin (Poland), has the form of a hexagonal array of air-holes with the holey-area limited to three concentric rings of the holes. The central hole of the periodic structure is missing, forming a solid glass core in the center of the fiber. Geometrical parameters of the fiber are as follows: the distance between neighboring holes $\Lambda=6.5 \mu \mathrm{m}$ and the holes diameter $\mathrm{d}=3.9 \mu \mathrm{m}$. It gives the diameter-to-pitch ratio (filling factor) of 0.6. As a guest material for the analyzed PLCF, a prototype NLC mixture, named 1550 [12], synthesized at the Military University of Technology and 
composed of alkyl 4-trans-4-trans-alkylcyclohexyl cyclohexylcarbonate was chosen. The temperature dependence of its refractive indices is presented in Fig. $1 \mathrm{~b}$.

Numerical simulations were performed by implementing the finite difference beam propagation method (FD-BPM) [13] for solving electromagnetic wave equation (obtained with an assumption of paraxial and slowly-varying envelope approximation) in the following form:

$$
2 \mathrm{ik}_{0} \mathrm{n}_{0} \frac{\partial \mathrm{E}}{\partial \mathrm{z}}=\nabla_{\perp}^{2} \mathrm{E}+\mathrm{k}_{0}^{2}\left(\mathrm{n}^{2}-\mathrm{n}_{0}^{2}\right) \mathrm{E},
$$

where $\mathrm{k}_{0}$ is a free-space wave vector, $\mathrm{n}_{0}$ is an effective refractive index, $\mathrm{z}$ is a propagation direction, $\mathrm{E}$ is a slowly-varying complex amplitude of the electric field, and $n=n(x, y, z)$ is a refractive index distribution in the fiber cross-section. The latter may depend on the optical power of injected light and in our case, a standard Kerrlike nonlinearity has been considered with a nonlinear refractive index: $\mathrm{n}_{\mathrm{NL}}=\mathrm{n}_{\mathrm{L}}+\mathrm{n}_{2} \mathrm{I}$, where $\mathrm{n}_{\mathrm{L}}$ is the linear refractive index (i.e. obtained for the low-power excitations), $\mathrm{n}_{2}$ is a nonlinear (Kerr) coefficient and $\mathrm{I}$ is the intensity of the propagating beam. It is worth noting that the analyzed scalar problem described by Eq. (1) was simplified by assuming that the initial NLC refractive index distribution within PCF holes is homogeneous. Moreover, by supposing that infiltration of PCFs by using the capillary effect results in the planar orientation of NLC molecules within fiber capillaries (with possible enhancement by special orientation treatments [14]), an ordinary refractive index $\mathrm{n}_{0}$ of NLC was applied in calculations. The values of $n_{0}=1.4583$ for the 1550 NLC at room temperature, and $\mathrm{n}_{\mathrm{g}}=1.4563$ for silica glass at a wavelength of $660 \mathrm{~nm}$ were used.

In numerical simulations performed a Crank-Nicolson scheme was applied with propagation step of $0.1 \mu \mathrm{m}$.

Whereas the ordinary refractive index of the considered NLC is greater than for silica glass (see Fig. $1 b)$, the effective refractive index of the PLCF cladding is higher than that of the fiber core. It means that light propagation within the core is possible only due to the photonic bandgap (PBG) effect. Otherwise, discrete light propagation (discrete diffraction) as an effect of tunneling between neighboring NLC-infiltrated PCF holes (from here on also called NLC rods/channels/waveguides) is observed, as illustrated in Fig. 2. In particular, Fig. 2 presents low-power light propagation for the Gaussian beam injected into the glass core of a PLCF structure with the results obtained for the square discrete mesh with a grid period of $\mathrm{h}=0.4 \mu \mathrm{m}$. It is worth noting that the limited size of the holey region of the PCF causes light to reach the border of the periodic structure and come back to the central part of the fiber. As a result, the light beam is alternately broadening and narrowing on the way of propagation (as seen in Fig. 2). A detailed look at the intensity distributions shown in Fig. 2 reveals their imperfections. Unsymmetrical beam broadening (narrowing) seen was still noticeable for numerical simulations performed with a twice denser mesh grid, i.e. $\mathrm{h}=0.2 \mu \mathrm{m}$ (not presented here).
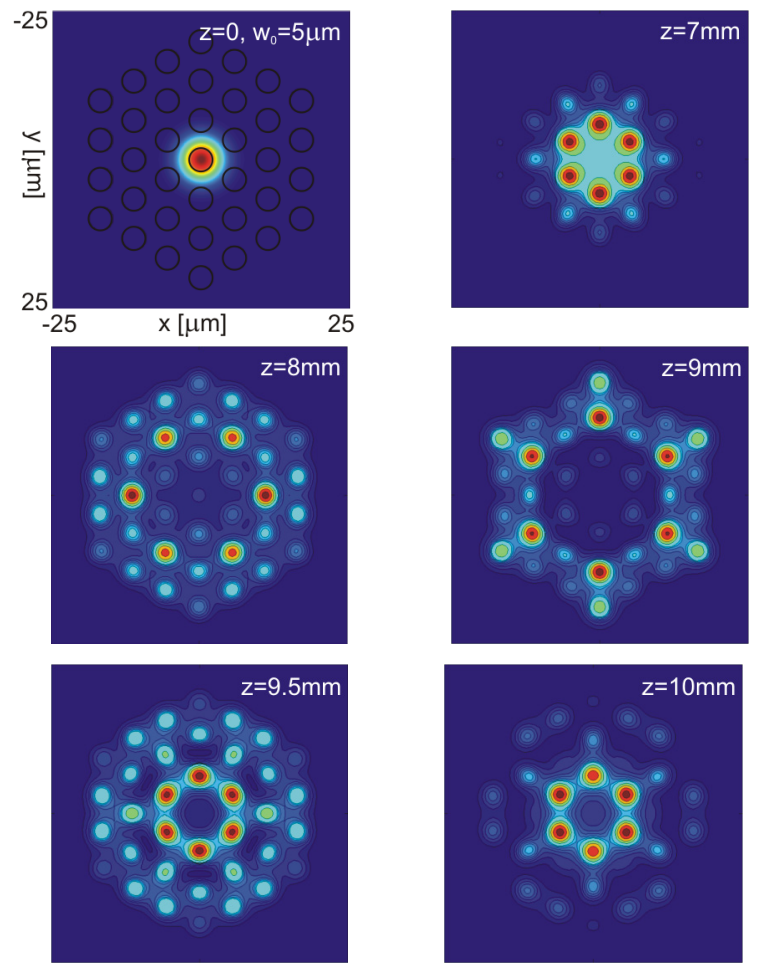

Fig. 2. Beam transverse intensity profiles (i.e. $\sim|E|^{2}$ ) along propagation direction z. Numerical results presented obtained for the square-shaped mesh with the nods separated by $\mathrm{h}=0.4 \mu \mathrm{m}$.

It has provoked additional analyses on uniform discrete mesh application in the FD-BPM algorithm. As it is well known, it is necessary to choose the proper grid shape and its resolution to get the best representation of a numerically reproduced photonic structure. It is also important to find the optimal grid form and its spacing that ensures the stability of numerical procedure with minimization of required computation time (keeping the required calculations precision). One of the possible approaches applied in the FD-BPM is to use the square grid with a resolution suited to the considered numerical problem. In PLCFs with the hexagonal symmetry analyzed here, even the application of a highly-dense square mesh does not help to avoid differences in distances between adjacent holes, along with their sizes and shapes obtained in the digitalization process. It is so mainly because of the incapability to have the center of each hole in a grid node when the square mesh is applied. The lack of symmetry in the numerical distribution of fiber holes and differences in hole sizes and shapes results in different coupling coefficients for the neighboring NLC rods. It means that numerically a 
periodical matrix of the NLC channels does not consist of identical elements. It causes asymmetrical beam intensity distribution in the fiber cross-section on the way of propagation (i.e. the diffractive divergence of a light beam launched into the fiber core is not the same in all radial directions even if the structure is completely symmetric).

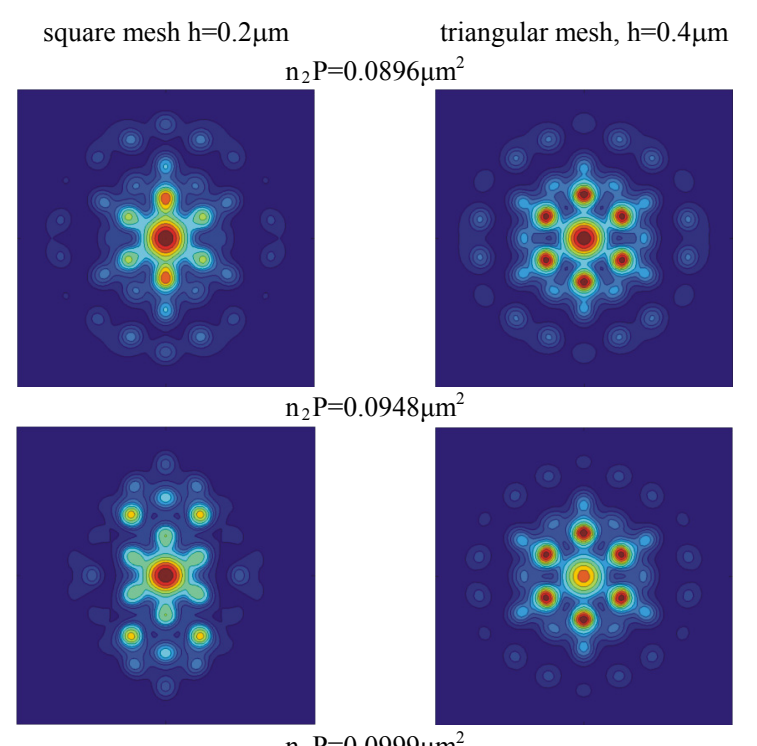

$\mathrm{n}_{2} \mathrm{P}=0.0999 \mu \mathrm{m}^{2}$

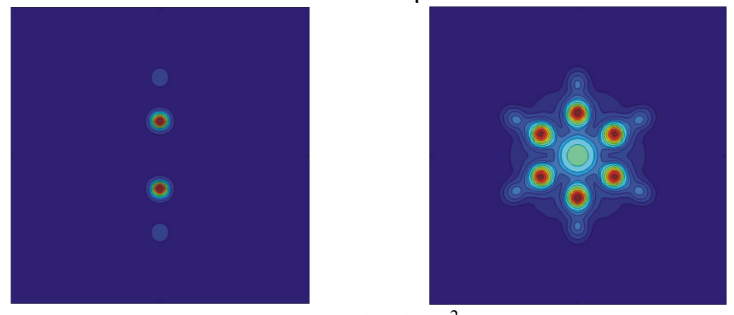

$\mathrm{n}_{2} \mathrm{P}=0.1050 \mu \mathrm{m}^{2}$
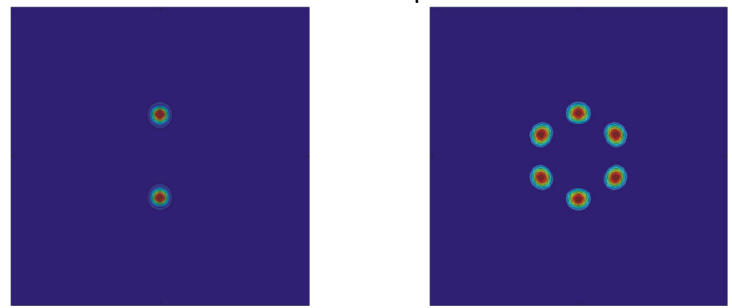

Fig. 3. Light intensity distribution for the propagation distance of $2.5 \mathrm{~mm}$ as a function of the input optical power $\mathrm{P}$ obtained for the square (left) and triangular (right) mesh. The $5 \mu \mathrm{m}$-waist Gaussian beam is initially launched into the glass core of the fiber and the initial refractive index of NLC (i.e. for $\mathrm{P} \sim 0$ ) was taken as $\mathrm{n}_{0}$. It is worth noting that for the Kerr coefficient $\mathrm{n}_{2}=10^{-10} \mathrm{~m}^{2} / \mathrm{W}$ the product $\mathrm{n}_{2} \mathrm{P}=0.1 \mu \mathrm{m}^{2}$ corresponds to an optical power of $1 \mathrm{~mW}$. The size of the numerical window presented is the same as in Fig. 2.

This problem exaggerates with the propagation distance and/or with optical power (see the left column of Fig. 3). When the power of a propagating beam increases, the refractive index of the excited NLC waveguides is modified by optical nonlinearity. Positive Kerr-like nonlinearity $\left(\mathrm{n}_{2}>0\right)$ analyzed here corresponds to reorientational nonlinearity observed in NLCs. In such a case, an increase in optical power results in stronger guiding of light within NLC rods, which may lead to light self-localization. If the input beam power is high enough, nonlinearity balances diffractive broadening and light propagates in a limited region of the fiber (here it is a first ring of the holes). The numerical results, showing the influence of focusing nonlinearity on light beam propagation, obtained for both: square and triangular mesh, are shown in Fig. 3. The problem of strong asymmetry of beam profiles in the case of a square mesh may be solved by applying a triangular grid. In such a case it is possible to have the center of each NLC rod in a grid node and to have equal distances between neighboring holes, as well as their sizes and shapes after numerical representation.

To conclude, we have presented numerical modeling of light propagation in a PLCF structure. The results of the simulations performed, based on the FD-BPM (with a Crank-Nicolson scheme applied), have demonstrated the advantages of triangular mesh application (especially in the case of high-power light propagation) for analysis of photonic crystal fibers with hexagonal symmetry.

The authors would like to acknowledge the "Master" programme granted by the Foundation for Polish Science. K.A.R thanks to Marie Curie Outgoing International Fellowship (MOIF-CT-2006-039600) for financial support.

\section{References}

[1] P. Russel, Science 299, 358 (2003).

[2] I.C. Khoo, S.T. Wu, Optics and Nonlinear Optics of Liquid Crystals (Singapore, World Scientific Publ., 1997).

[3] T.T. Larsen, A. Bjarklev, D.S. Hermann, J. Broeng, Opt. Expr. 11, 2589 (2003)

[4] T.R. Woliński, K. Szaniawska, K. Bodnarczuk, P. Lesiak, A.W. Domański, R. Dąbrowski, E. Nowinowski-Kruszelnicki, J. Wojcik, Opto-Electron. Rev., 13(2), 177 (2005).

[5] M.M. Tefelska, M.S. Chychłowski, T.R. Woliński, R. Dąbrowski, J. Wójcik, Phot. Lett. of Poland 1(2), 97 (2009).

[6] A. Czapla, W.J. Bock, T.R. Woliński, Phot. Lett. of Poland 1(2), 100 (2009).

[7] T.R. Woliński, K. Szaniawska, S. Ertman, P. Lesiak, A.W. Domański, R. Dabrowski, E. Nowinowski-Kruszelnicki, J. Wojcik, Meas. Science Techn. 17, 985 (2006).

[8] K.A. Brzdąkiewicz, U.A. Laudyn, M.A. Karpierz, T.R. Woliński, J. Wójcik, Opto-Electron. Rev. 14, 287 (2006).

[9] K.A. Rutkowska, U.A. Laudyn, R.T. Rutkowski, M.A. Karpierz, T.R. Woliński, J. Wójcik, Proc. SPIE 6582, 658215 (2007).

[10] M. Kwaśny, U.A. Laudyn, P. Jung, M.A. Karpierz, Phot. Lett. of Poland 1(4), 160 (2009).

[11] I.H. Molitson, J. Opt. Soc. Am. 55, 1205 (1965).

[12] J. Schirmer, P. Kohns, T. Schmidt-Kaler, A. Muravski, S. Yakovenko, V. Bezborodov, R. Dabrowski, P. Adomenas, Mol. Cryst. Liq. Cryst. 307, 1 (1997).

[13] A.R. Mitchell, D.F. Griffiths, The Finite Difference Method in Partial Differential Equations (London, Wiley 1980).

[14] S. Ertman, T.R. Woliński, A. Czapla, K. Nowecka, E. Nowinowski-Kruszelnicki, J. Wójcik, Proc. SPIE 6587, 658706 (2007). 